\title{
LOCATING REPLACEMENT AZIMUTH SCREWS FOR TASCAM COMPACT CASSETTE DECKS
}

Andrew Thomas, CHL AV Preservation, USA

Audio cassette tapes were widely used for capturing audio events. Many archives are subsequently digitizing extensive cassette tape collections. One of the most important steps when capturing a cassette tape is proper adjustment of the azimuth screw, which allows you to capture the full range of recorded frequency content.

In audio terms, "azimuth" refers to the measure of the angle between the tape head gap and the physical tape itself. Because the angle of the head gap can vary from machine to machine, it is essential to adjust the azimuth for each tape captured for preservation. Failure to adjust the playback head azimuth may result in dull sounding audio. The spectrographic images below illustrate the frequency spectrum from two captures of the same cassette tape. The image on the left shows the limited frequency range before the azimuth screw was adjusted. The image on the right shows the broadening of the frequencies after azimuth adjustment. Clearly, proper azimuth adjustment is essential to preserving the full range of audio.

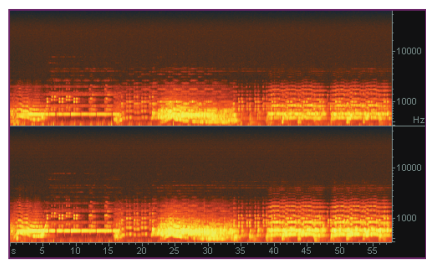

Before Azimuth Adjustment

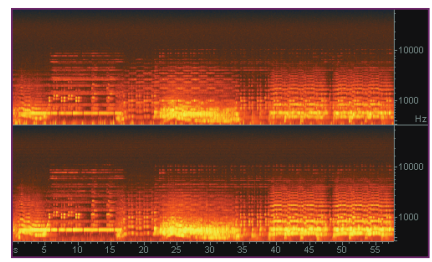

After Azimuth Adjustment

The Tascam I22MKIII cassette tape deck is a favorite deck for playback of cassette tapes by many archives. They are well built machines that have great features like smooth tape handling mechanisms, precise manual bias and level adjustment on the front panel, and balanced XLR inputs and outputs. We've had good experiences with these decks and have found them to be well suited for performing archival transfers. But like any mechanical device, parts wear out and need to be replaced. Because the azimuth screw is adjusted frequently, it tends to wear fast and require replacement. The head of the screw can easily become so stripped that it is almost impossible to adjust. This problem has occurred on every cassette deck that we own (most of which are Tascam 122 models).

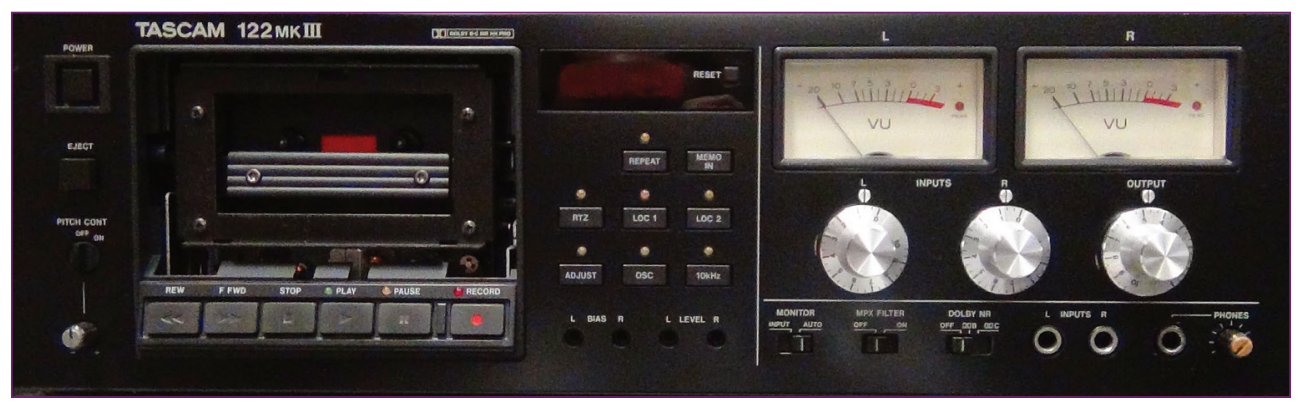

Tascam I22 MKIII Cassette Deck

Once we were down to only a few machines that didn't have stripped azimuth screws, we decided to try and track down a replacement. We believed this would be a fairly easy task; but were sadly unsuccessful until recently. We decided to write this short article so others wouldn't have to endure the seemingly endless loop of buying numerous screws only to realize they wouldn't work and then having to sending them back. 


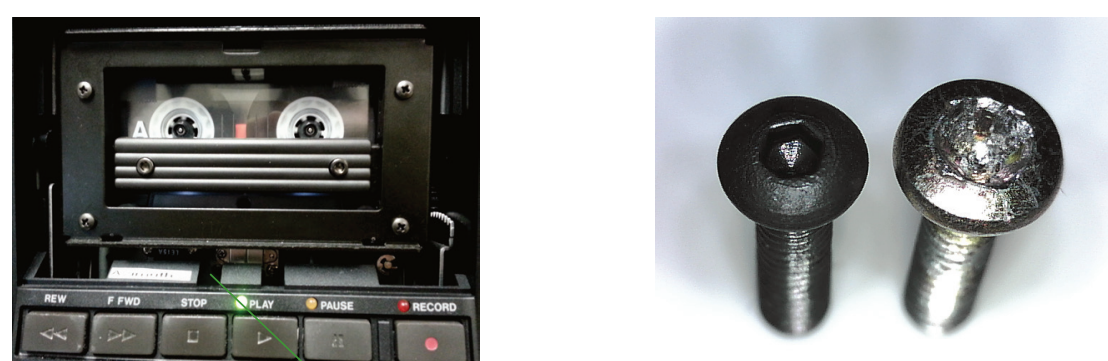

New Screw / Stripped Screw

Azimuth screw location on a Tascam I22 MKIII

When the correct replacement was finally found, we also decided to replace the Phillips screw head with a hex head screw. The hex head being less likely to become "stripped" during regular use.

This screw size works with Tascam models I I 2 MKII, I 22 MKII / MKIII, and I 34. This screw size might work with other decks, but we have not checked. The azimuth screw size is the following:

\section{M2-0.40x8 MM (Metric) \\ I 2.9 ISO 7380}

We hope this is helpful in your pursuit of optimal sound. 